\title{
Claudin 6: Therapeutic prospects for tumours, and mechanisms of expression and regulation (Review)
}

\author{
HUAN DU ${ }^{1,2}$, XIYUE YANG ${ }^{2}$, JINJIA FAN $^{2}$ and XIAOBO DU ${ }^{1}$ \\ ${ }^{1}$ Department of Oncology, Mianyang Central Hospital, Mianyang, Sichuan 621000; ${ }^{2}$ Department of Oncology, \\ Affiliated Hospital of North Sichuan Medical College, Nanchong, Sichuan 637000, P.R. China
}

Received November 1, 2020; Accepted June 30, 2021

DOI: $10.3892 / \mathrm{mmr} .2021 .12316$

\begin{abstract}
Tight junctions (TJs) are an important component of cell connectivity; they maintain cell polarity, permeability and adhesion, and participate in the regulation of cell proliferation and differentiation. The claudin (CLDN) family is integral to TJs, and CLDN6 is an important member of this family. Abnormal expression of CLDN6 can destroy the integrity of TJs through various mechanisms and can serve multiple roles in the occurrence and development of tumours. CLDN6 is widely expressed in various tumours but rarely expressed in healthy adult tissues. The aim of this review is to critically examine the recent literature on CLDN6, including its structure, expression in different tumours, regulatory mechanisms and therapeutic prospects. Although some conclusions are controversial, in certain tumours, such as liver, ovarian, endometrial and oesophageal cancer, and atypical teratoid/rhabdoid tumours, research consistently shows that CLDN6 is expressed in tumour tissues but is not expressed or is expressed at low levels in surrounding tissues. In these tumours, CLDN6 has potential as a carcinoembryonic antigen and a therapeutic target.
\end{abstract}

\section{Contents}

1. Introduction

2. Structural characteristics of CLDN6

3. Expression and regulation mechanisms of CLDN6 in tumours

4. CLDN6 and drug resistance in cancer

5. Prospect of CLDN6 in the treatment of tumours

6. Discussion

Correspondence to: Dr Xiaobo Du, Department of Oncology, Mianyang Central Hospital, 12 Changjiaxiang Mianyang, Sichuan 621000, P.R. China

E-mail: duxiaobo2005@126.com

\section{Abbreviations: CLDN6, claudin 6; TJs, tight junctions}

Key words: review, CLDN6, target, cancer, structure, expression, regulatory mechanisms, therapeutic prospects

\section{Conclusion}

\section{Introduction}

The monolayer epithelium is the largest tissue lining in a number of human organs, and its integrity is maintained by the cell junction complex. Tight junctions (TJs) exist in the junctional complexes of epithelial and endothelial cells, and serve crucial roles in cell polarity, adhesion and permeability (1). TJs also maintain the integrity of the tissue structure and participate in transmembrane movement and signal transduction inside and outside of cells (2). A decrease in TJ integrity and an increase in cell bypass permeability are characteristics of tumours and inflamed tissues (3). TJs also have adhesive properties and can prevent the shedding of epithelial cells. The initial stage of tumour metastasis is a disconnect between tumour and endothelial cells. Therefore, TJs are the first obstacle that must be overcome in tumour metastasis (4). The decrease in TJ integrity leads to the perfusion and extravasation of cancer cells through the endothelial barrier (5). TJs are composed of three basic membrane proteins: Occludin, claudin (CLDN) and junction adhesion molecules. The CLDN protein family serves a key role in the functions of TJs (6) (Fig. 1), including helping regulate defence and barrier functions, and differentiation and polarity in epithelial and endothelial cells (7). Tumour progression is characterized by the migration, invasion and metastasis of cancer cells. CLDNs are believed to serve a significant role in these processes as their loss contributes to the loss of cell junctions in a tissue-dependent manner. CLDN expression can affect cancer progression in several ways: First, changes in CLDN expression cause $\mathrm{TJ}$ disorder and leakage, which is conducive to tumour metastasis and invasion; second, a decrease in cell polarity increases the supply of nutrition and growth factors to the tumour, and increases tumour cell expansion; and third, a decrease in intercellular adhesion may increase the risk of metastasis and promote tumour invasion (8). The CLDN family forms homologous and heterologous interactions between adjacent cells and is a key regulator of tumorigenesis and metastasis. Classic CLDNs (CLDNs 1-10, 14, 15, 17, and 19) also exhibit a much stronger sequence homology than the non-classic CLDNs (CLDNs 11-13, 16, 18, and 20-27) (9) (Fig. 2). Recent findings have shown that abnormal expression of CLDNs is closely related to the occurrence, progression and prognosis of tumours (10). Specifically, the CLDN family member CLDN6 
is expressed in numerous tumours but rarely found in adult normal tissues (11). Although some studies have reported results that contradict this, the high expression of CLDN6 in certain tumours makes CLDN6 a potential therapeutic target in these tumour types. Therefore, the present study reviewed the literature on CLDN6, including information on its structure, expression in different tumours, regulatory mechanisms and therapeutic prospects.

\section{Structural characteristics of CLDN6}

CLDN6 is one of 27 members of the CLDN family; it is located on chromosome $16 \mathrm{p} 13.3$ and has a molecular weight of $23 \mathrm{kDa}$ (12). CLDN6 has four transmembrane domains and a PDZ-binding region at the carboxyl end of the cytoplasm. This CLDN can bind with signal proteins and cytoskeletal proteins, and participate in the cellular response to external and intracellular signal transmission (13). CLDN6 is the only CLDN to potentially show specificity, as it can activate cell adhesion signals and regulate the activity of nuclear receptors (14). CLDN6 is expressed in a variety of embryonic epithelia, induces epithelial cell junction formation and polarity, and participates in the differentiation of stem cells into epithelial cells. Overall, CLDN6 is an important component of the CLDN family and serves a substantial role in maintaining the function of TJs (15).

\section{Expression and regulation mechanisms of CLDN6 in tumours}

Previous findings have shown that changes in CLDN expression cause TJ disorders and leakage $(16,17)$. This decrease in cell polarity increases the supply of nutrition and growth factors for tumour cells. In addition, the decrease in intercellular adhesion may increase the metastatic potential (18). Previous findings have shown that as a member of the CLDN family, CLDN6 is strongly expressed in the foetal stomach, lung and kidney, but not in healthy adult tissues $(19,20)$. However, other studies have not reached the same conclusion $(21,22)$, and the specific expression and regulatory mechanisms for CLDN6 remain unclear or controversial. Regardless, it is accepted that CLDN6 is expressed in a variety of tumours and serves an important role in the occurrence and development of tumours. The known expression and regulatory mechanisms of CLDN6 in various tumour types are shown in Table I.

Breast cancer. CLDN6 cannot be detected in human breast cancer cell lines and tissues or is expressed at lower levels than in healthy breast tissue $(21,22)$. In addition, inhibiting CLDN6 expression leads to an increase in the resistance of tumour cells to various types of apoptosis, enhancement of anchoring independent growth characteristics and promotion of breast cancer progression. Conversely, overexpression of CLDN6 restores the integrity of TJs, inhibits the epithelial mesenchymal transition (EMT) of breast cancer cells, inhibits the proliferation of breast cancer cells, decreases the migration and invasion of tumour cells, and induces the apoptosis of tumour cells (23). Knocking out CLDN6 can eliminate the inhibition of EMT-related genes and promote cell migration and invasion. In addition, the expression of CLDN6 is independently associated with BRCA1-related breast cancer (24). Intratumoral overexpression compared with the healthy surrounding tissue was significantly more frequent in BRCA1 mutation carriers for CLDN6. However, compared with all sporadic breast cancer cases, the incidence of breast cancer with low CLDN expression (CLDN-low; E-cadherin and CLDN3, 4, and 7) breast cancer was significantly higher among BRCA1 mutation carriers (23). Finally, CLDN6 can act as a tumour suppressor, and upregulating the expression of CLDN6 may be helpful in preventing breast cancer (17).

There are numerous studies on the role of CLDN6 in breast cancer (25), which involves different pathways (Fig. 3).

TGF- $\beta /$ Smad pathway. It has been suggested that silencing CLDN6 may be related to DNA methylation mediated by DNA methyltransferase 1 (DNMT1), which is regulated by the TGF- $\beta /$ Smad pathway, and thereby has an effect on the invasion and metastasis of breast cancer cells (26).

Methyl CpG binding protein 2 (MeCP2)/DNMT1/5-aza2-deoxycytidine (5-aza-dC)/trichostatin A (TSA) pathway. Li et al (27) hypothesized that DNA methylation of CLDN6 promotes the migration and invasion of breast cancer cells through the recruitment of MeCP2 and deacetylation. In one study, a methyltransferase inhibitor (5-aza-dC) and a histone deacetylase inhibitor, TSA, acted synergistically and induced the expression of CLDN6 in breast cancer cells (28). In addition, 5-aza-dC treatment can induce the demethylation of CLDN6, inhibit the binding of MeCP2 and the CLDN6 promoter, ease the chromatin structure of the CLDN6 gene and upregulate the expression of CLDN6 in breast cancer cells, thereby inhibiting the migration and invasion of tumour cells. CLDN6 silencing restores the migration and invasiveness of tumour cells (28).

MAPK/MMP2 pathway. As reported by Ren et al (29), CLDN6 gene silencing may promote the proliferation and migration of human breast epithelial cells through the MAPK pathway and increase the activity of MMP2. Moreover, the inhibition of CLDN6 expression may enhance the resistance of tumour cells to apoptosis, leading to tumour progression.

Hypoxia-inducible factor $1 A(H I F-1 \alpha)$ pathway. Due to the rapid growth of tumours and abnormalities in the tumour vascular system, hypoxia is prevalent in breast cancer. Therefore, HIF-1 $\alpha$ is closely related to breast cancer metastasis (22). Researchers discovered a negative feedback loop between CLDN6 and HIF-1 $\alpha$, in which HIF-1 $\alpha$ increased and upregulated CLDN6 expression during hypoxia. After overexpression of CLDN6, negative feedback inhibited the expression of HIF-1 $\alpha$ to decrease the metastasis of breast cancer (29). The downregulation of CLDN6 expression was positively correlated with lymph node metastasis in breast cancer (30).

Apoptosis signal regulated kinase 1 (ASK1)-p38/c-Jun $N$-terminal kinase (JNK) pathway. In a previous study, it was found that CLDN6 gene expression was related to the expression level of ASK1 (31), which induces apoptosis of breast cancer cells by regulating the ASK1-p38/JNK signalling pathway. 


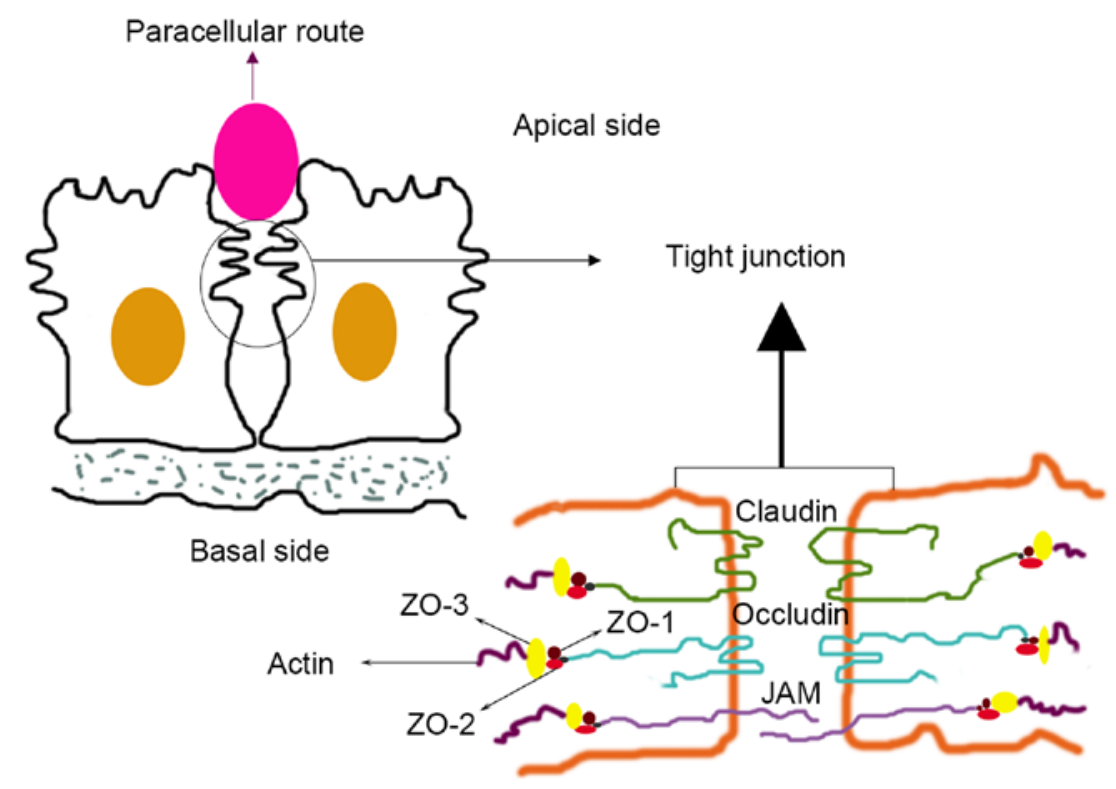

Figure 1. Structural diagram of tight connection. Upper panel represents the location diagram of the TJ. The lower panel is the schematic diagram of the main components of TJs. Pink oval, paracellular route. TJ, tight junction. JAM, junctional adhesion molecule; ZO-1, Zona Occludens 1.
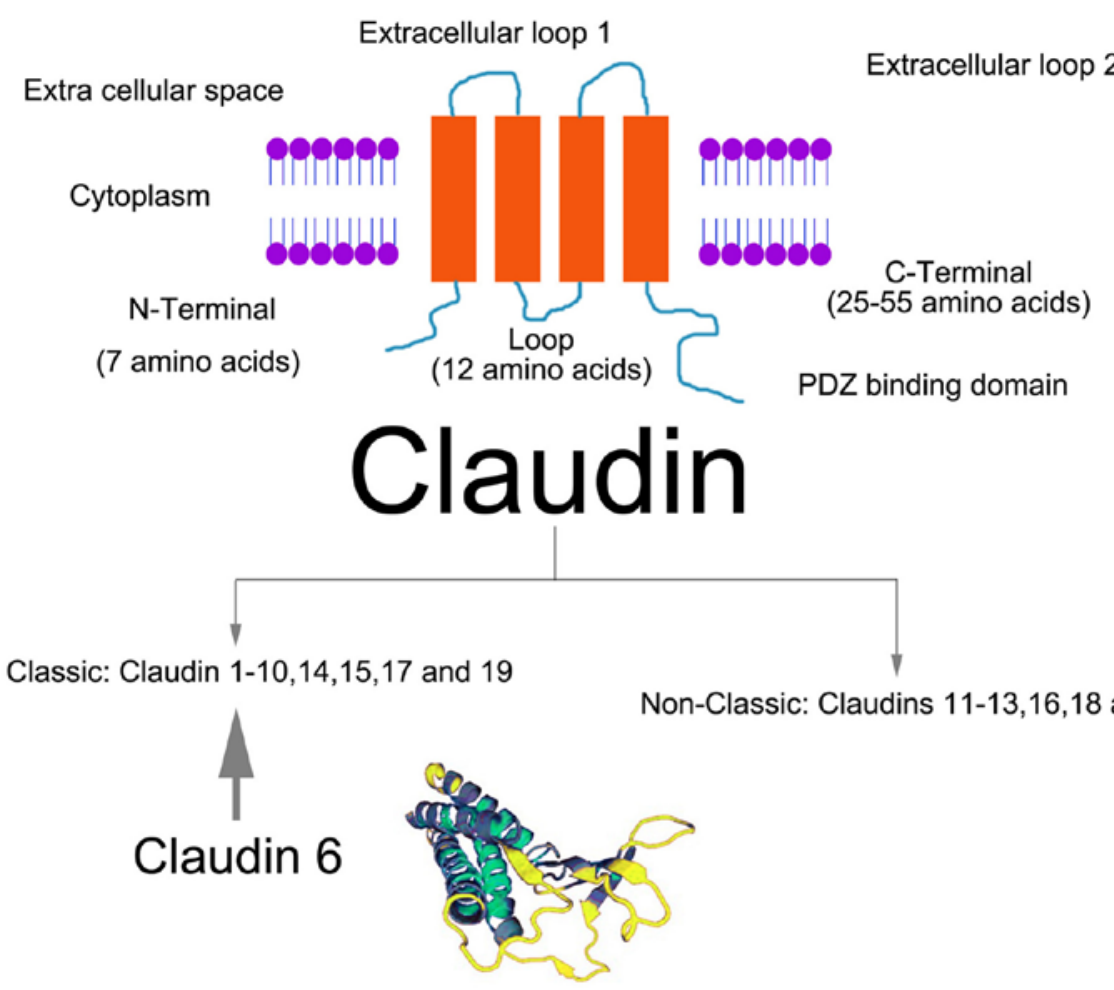

Figure 2. Structure diagram of CLDNs and molecular diagram of CLDN6. Upper panel shows the structure of CLDN proteins (monomer) and their classification based on their homologous sequences. The lower panel shows the molecular structure of CLDN6. CLDN, claudin; CLDN6, claudin 6.

ASK1 is a mitogen-activated protein kinase, which is involved in the activation of the JNK and p38 pathways. The expression of CLDN6 in breast cancer cells decreases the phosphorylation of ASK1, induces the activation of downstream target proteins JNK and p38 kinase, and leads to tumour cell death (32).

Oestrogen receptor $\beta($ Er $\beta) / E r \alpha-B e c l i n 1$ pathway. Oestrogen has been found to regulate the expression of CLDN6 at the transcriptional level through $\operatorname{Er} \beta$ (33). $\operatorname{Er} \beta$ can induce autophagy in tumour cells through CLDN6 and inhibit the migration and invasion of breast cancer cells. In addition, Beclin1 is a key regulator of autophagy, and the expression of Er $\beta$ and CLDN6 in breast cancer has been positively correlated with Beclin1 expression. The prognosis of patients with breast cancer with high expression of Er $\beta$, CLDN6 and Beclin1 was improved compared with that of patients with low expression 
Table I. Expression of claudin 6 in human tumours.

\begin{tabular}{lcc}
\hline & \multicolumn{2}{c}{ (Refs.) } \\
\cline { 2 - 3 } Cancer type & Cell & Tissue \\
\hline Breast invasive carcinoma & $(17,25-35,65,66)$ & $(21-24)$ \\
Gastric cancer & $(37,39,41,43-45)$ & $(36,38-40,42)$ \\
Hepatocellular carcinoma & $(49)$ & \\
Cervical carcinoma & $(50)$ & $(51)$ \\
Non-small cell lung cancer & $(52)$ & $(53)$ \\
Ovarian cancer & & $(54)$ \\
Endometrial carcinoma & $(55)$ & \\
Atypical teratoid/rhabdoid & & $(56-58)$ \\
tumours & & \\
Oesophageal squamous & & \\
cell carcinoma & & \\
\hline
\end{tabular}

of these proteins (33). Knocking out the Beclin1 gene can also reverse the autophagy induced by CLDN6 and the inhibition of CLDN6 on breast cancer metastasis (34). Wu et al (35) also suggested that the expression of CLDN6 in breast cancer cells is regulated by the $\mathrm{ER} \alpha$ pathway, and that upregulation of CLDN6 expression may be helpful in preventing breast cancer.

Gastric cancer. One report indicated that the expression of CLDN6 differed between gastric cancer and adjacent tissues (36). Additional studies showed that CLDN6 expression differed in gastric cancer compared with healthy tissue (37-41), and according to Zavala-Zendejas et al (37), the expression of CLDN6 in gastric cancer tissues was higher compared with that in adjacent tissues. CLDN6 is a gene promoter in gastric cancer, and its upregulation enhances the tumorigenicity of gastric cancer cells (37). Several studies reported that the upregulation of CLDN6 expression was positively correlated with a decrease in overall survival rate in gastric cancer, while CLDN6 silencing inhibited the proliferation and invasion of tumour cells $(39,42)$.

However, according to Gao et al (39), the expression level of CLDN6 in gastric cancer tissues was lower compared with that in adjacent tissues. Results of the study showed that the expression level of CLDN6 was associated with age, lymph node metastasis, pathological stage and distant metastasis. The expression of CLDN6 in patients $<50$ years of age was higher compared with that in patients $>50$ years of age. Furthermore, the survival rate of patients with high expression of CLDN6 was significantly higher compared with that of patients with low CLDN6 expression (40). Decreasing the expression of CLDN6 may destroy the integrity of epithelial cells, leading to paracellular leakage, an increased nutrient supply and promotion of metastasis (41) (Fig. 4).

An additional regulatory role for CLDN6 involves the large tumour suppressor kinase (LATS)1/2/yes-associated protein 1 (YAP1) pathway and intratumoral heterogeneity (ITH) gene mutation. CLDN6 may promote the proliferation and invasion of gastric cancer by affecting the Yap1 and Yap1-snail axes (43). The interaction between CLDN6 and LATS1/2 in the Hippo signalling pathway decreases the phosphorylation of LATS1/2 and Yap1, affects Yap1 entry into the nucleus, changes target gene modification, affects the EMT process and enhances the invasion ability of gastric cancer cells (43). CLDN1 is a pre-MMP2 activator, while CLDN6 promotes the migration and invasion of gastric cancer cells by promoting the expression of CLDN6 and inducing the activation of MMP2 (44). Previous findings have shown that CLDN6 may change TJ function in gastric cancer through gene mutations and in this way may promote the occurrence of cancer. The CLDN6 gene contains not only a frameshift mutation, but also an ITH mutation (44). Previous findings have shown that lipopolysaccharides from Helicobacter pylori can increase the expression of CLDN-4, $-6,-7$ and -9 in gastric cancer cells by inducing Toll-like receptor TLR2 expression (45). Overall, the expression of CLDN6 in gastric cancer is associated with a poor prognosis and a short survival period (45).

Hepatocellular carcinoma (HCC). HCC is one of the most common malignant tumours of the digestive system, with high incidence and mortality rates. In particular, China has a high incidence of hepatitis B and liver cancer (46). Therefore, it is important to identify potential therapeutic targets for HCC. CLDN6 is highly expressed in HCC and is also expressed in the liver during hepatitis $\mathrm{C}$ virus (HCV) replication. CLDN6 is the receptor for $\mathrm{HCV}$ and is therefore necessary for the entry of HCV into cells $(47,48)$. In addition, CLDN6 can promote EMT and enhance the proliferation and invasion of hepatoma cells. Moreover, the upregulation of CLDN6 expression may act as an oncogene in HCC through the EGFR/Akt/mTOR signalling pathway, and may promote the proliferation, metastasis and invasion of HepG 2 cells. By contrast, CLDN6 silencing inhibits the proliferation and metastasis of hepatoma cells (49).

Cervical carcinoma. CLDN6 expression was downregulated in cervical cancer cells $(50,51)$, which is inconsistent with the conclusion of Reinhard et al (11). Zhang et al (50) hypothesised that the occurrence of cervical cancer may be related to the decreased expression of CLDN6 and that increased expression of CLDN6 may inhibit the proliferation of tumour cells (51). CLDN6 may also promote the apoptosis of cervical cancer cells by inhibiting the activity of the AKT signalling pathway. Similarly, it may reverse EMT and inhibit the invasion of cervical cancer cells by binding to AF-6, thereby inhibiting the ERK signalling pathway (51). In this way, CLDN6 may act as a tumour suppressor gene in cervical cancer, and loss of CLDN6 may enhance the carcinogenicity of cervical cancer cells (50).

Non-small cell lung cancer (NSCLC). CLDN6 protein levels showed significant positivity in NSCLC cells, but were negative in normal lung cells and bronchial epithelial cells (52). In this study, the expression of CLDN6 was related to lymph node metastasis and Tumour-Node-Metastasis stage. However, in the study by Wang et al (53), the expression of CLDN6 in adjacent tissues was higher compared with that in NSCLC tissues. The survival rate of patients with low CLDN6 expression was significantly lower compared with that of patients with high CLDN6 expression. In addition, 


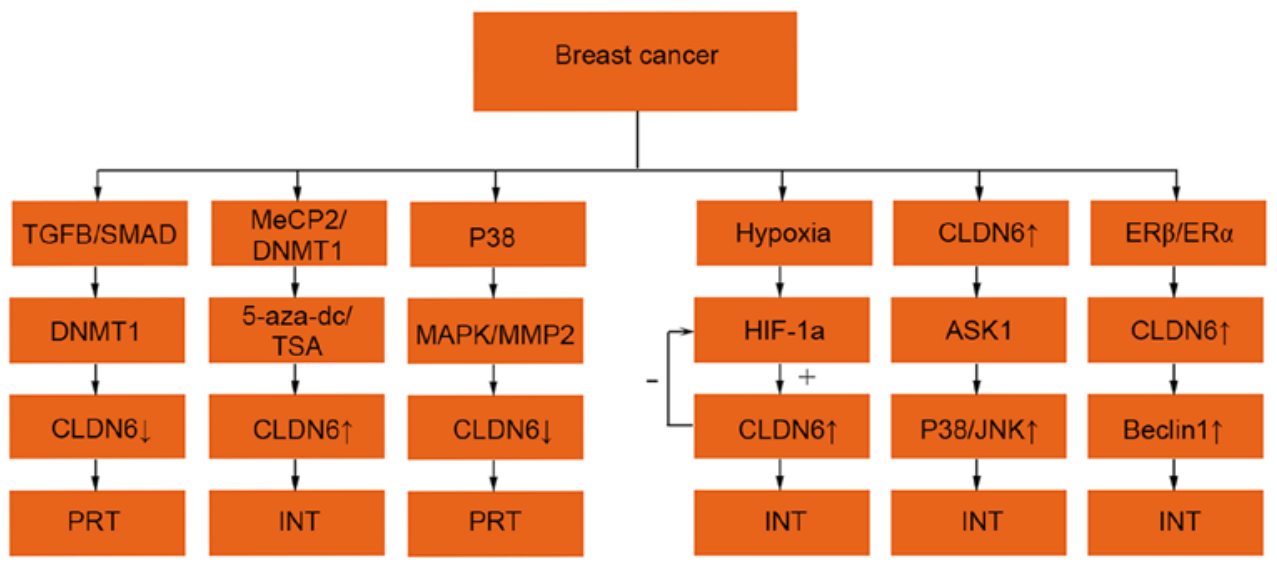

Figure 3. Regulatory mechanisms of CLDN6 expression in breast cancer. The expression $\uparrow$ is increased, $\downarrow$ decreased, + is promoting effect and -is inhibiting effect. CLDN6, claudin 6; TGF- $\beta$, transforming growth factor- $\beta$; MeCP2, methyl cytosine binding protein-2; DNMT1,DNA methyltransferase 1; 5-aza-dc, 5-aza-2-deoxycytidine; AKT, protein kinase B; MAPK, mitogen-activated protein kinase; ASK1, apoptosis signal-regulated kinase 1; MMP2, matrix metalloproteinase 2; HIF-1 $\alpha$, hypoxia inducible factor-1 $\alpha$; SMAD, small mothers against decapentaplegic; mall mothers against decapentaplegic; JNK, c-Jun $\mathrm{N}$-terminal kinase; PRT, promote tumour progression; INT, inhibition of tumour progression.

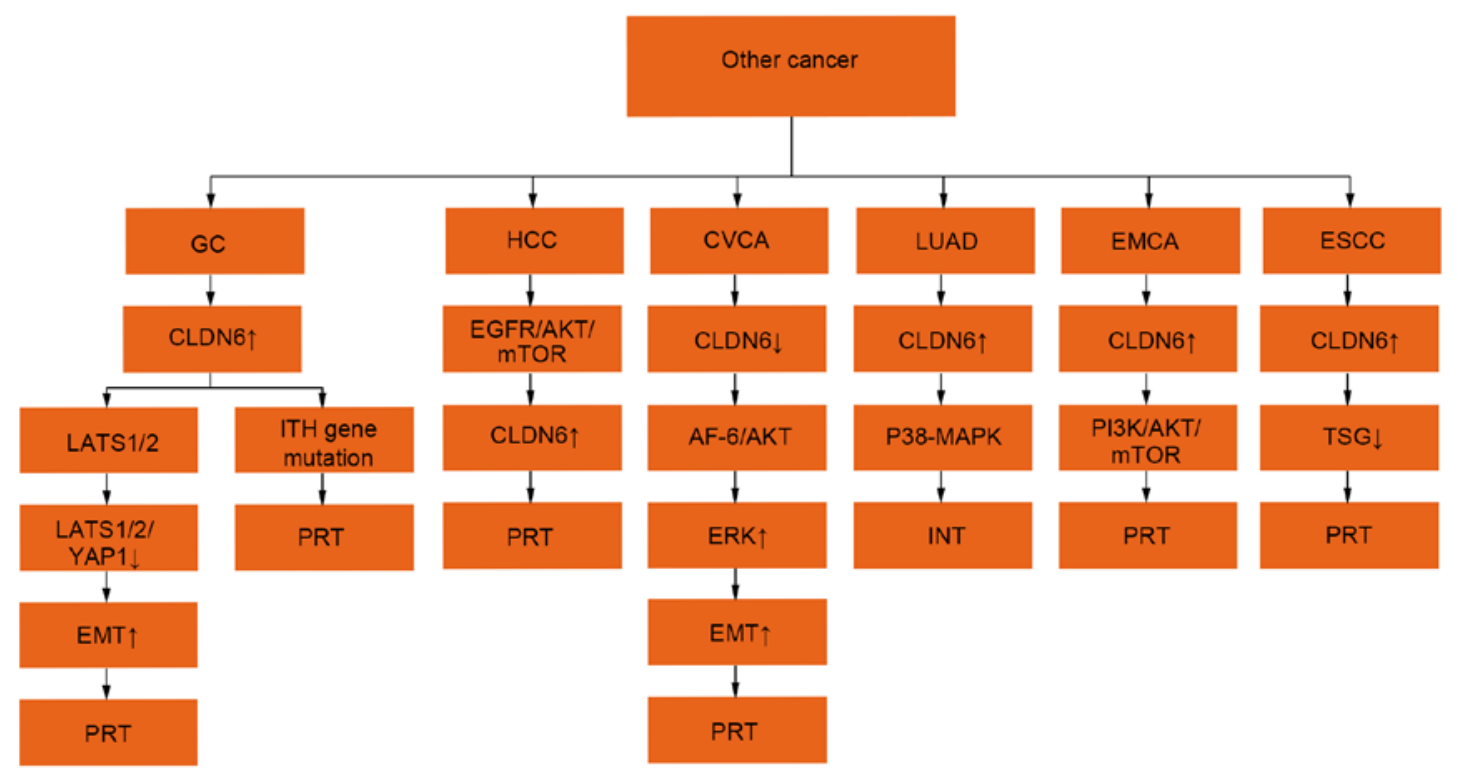

Figure 4. Regulatory mechanisms of CLDN6 expression in other tumours. The expression $\uparrow$ is increased, $\downarrow$ decreased, + is promoting effect and -is inhibiting effect. CLDN6, claudin 6; LATS1/2, large tumor suppressor 1 and 2; EMT, epithelial mesenchymal transition; YAP1, Yes-associated protein1; PI3K, phosphatidylinositol 3-kinase; MAPK, mitogen-activated protein kinase; ERK, extracellular regulated protein kinases; AKT, protein kinase B; EGFR, epidermal growth factor receptor; mTOR, mammalian target of rapamycin; ITH, intratumoral heterogeneity; AF-6, Protein AF-6; TSG, tumour suppressor gene; PRT, promote tumour progression, INT, inhibition of tumour progression.

low CLDN6 expression may be associated with poor prognosis in patients with NSCLC (53).

Ovarian cancer. CLDN6 was highly expressed in ovarian cancer tissues and showed low expression in normal ovarian epithelium. The high expression of CLDN6 in ovarian cancer cells suggests promotion of ovarian cancer cell proliferation. In addition, high CLDN6 expression enhanced cell migration and invasion abilities, and inhibition of the apoptosis of ovarian cancer cells. CLDN6 may serve an active role in the invasion and metastasis of ovarian cancer (54).

Endometrial cancer. The expression of CLDN6 was upregulated in endometrial carcinoma. CLDN6 gene knockout may inhibit the proliferation and migration of endometrial carcinoma HEC-1-B cells through the PI3K/Akt/mTOR signalling pathway. The expression of CLDN6 may be related to the overall survival rate of patients with endometrial carcinoma (55).

Atypical teratoid/rhabdoid tumours (AT/RTs). AT/RTs are highly aggressive brain tumours in children (56). CLDN6 was moderately or highly expressed in AT/RTs (57), but the expression level of CLDN6 was not associated with survival rate or clinical behaviour (58).

Oesophageal squamous cell carcinoma. The methylation frequency of CLDN6 in oesophageal carcinoma was higher 
compared with that in non-invasive tissues. Methylation of CLDN6 can result in the silencing of tumour suppressor genes and the development of oesophageal cancer (59). According to Tsunoda et al (59), CLDN6 may silence tumour suppressor genes through high-frequency methylation, thus promoting the occurrence and development of oesophageal cancer.

\section{CLDN6 and drug resistance in cancer}

Previous findings have shown that cancer stem cells (CSCs) are responsible for cancer treatment failure and drug resistance, and that CSCs serve an important role in tumour recurrence, metastasis and drug resistance. Therefore, understanding the factors that regulate CSCs has become very important $(60,61)$. In mouse stem cells, CLDN6 may trigger epithelial morphogenesis, while the expression of CLDN6 constitutes an early marker of embryonic stem cells $(62,63)$. Therefore, it is possible that CLDN6 may affect the biological functions of CSCs.

Chemotherapy is one of the primary treatments for breast cancer, and chemotherapy resistance is the main obstacle in breast cancer treatment (64). In a previous study (65), the expression of CLDN6 was significantly higher in the MCF-7/MDR cell line than in the MCF-7 cell line. The high expression of CLDN6 in MCF-7/MDR cells increased the resistance to Adriamycin (ADM), 5-fluorouracil and cisplatin, while the silencing of CLDN6 enhanced the killing effect of those drugs on the MCF-7/MDR cell line. It is also possible that CLDN6 is involved in the regulation of drug resistance in human breast cancer cells. The mechanism may be through CLDN6 regulating glutathione S-transferase $\pi 1$ (GSTP1) to promote the chemotherapeutic resistance of MCF-7 cells through p53, as GSTP1 expression and enzyme activity is regulated by $\mathrm{p} 53$. In cells that overexpress MCF-7, the expression of GSTP1 was upregulated (65). Furthermore, CLDN6 interacts with p53 to inhibit the transfer of p53 from the cell nucleus to the cytoplasm, leading to drug resistance. CLDN6 can also enhance the drug resistance of breast cancer cells to ADM by activating the AF-6/ERK signalling pathway and upregulating the characteristics of breast cancer cells (66). These findings may provide a new target and strategy for cancer treatment.

\section{Prospect of CLDN6 in the treatment of tumours}

In the study by Reinhard et al (11), CLDN6 was specifically expressed in a variety of tumours but not in healthy tissues. Although a number of research conclusions contradict this $(39,50,51)$, CLDN6 still has the potential to act as a tumour gene target in cancer types, such as liver, ovarian and endometrial cancer, and to provide new strategies for tumour treatment. The treatment modalities for this include antibody drug-binding targets, radionuclide therapeutic targets and new antibody therapeutic targets, among others.

A new patent for a monoclonal antibody against CLDN6 has been shown to be effective against testicular germ cell tumours (TGCTs). The use of CLDN6 antibody can block tumour growth and prolong the life of patients with TGCT. The presence of tumour-associated antigens can induce strong and persistent antigen-specific T-cell responses, break the immune system tolerance to endogenous self-antigens and produce effective antitumour effects (67).

Antibody drug conjugates (ADCs) are monoclonal antibodies that bind to cytotoxic drugs (68). The antibodies used by ADCs have high specificity for tumour cell surface proteins and can specifically kill tumour cells. ADCs can transport drugs to tumour sites through targeted drug delivery and decrease the toxicity of cytotoxic drugs to normal tissues $(69,70)$. As CLDN6 is a specific antigen found in some tumours, it may be a potential target for ADC drugs.

Targeted radionuclide therapy (TRT) uses a molecular carrier with a high affinity for tumour cell surface antigens to transport nuclides to tumour cells and sites of metastasis for treatment. Drugs containing radionuclides are targeted to accumulate in tumour tissues, which directly affects cancer cells and avoids damaging normal cells (71). TRT combines the specificity of molecular targeting and the cytotoxicity of ionising radiation to reduce toxicity to healthy tissues and provides a more effective tumour treatment method (72). This may be an important direction for tumour treatment in the future. As a specific antigen in some tumours, CLDN6 may be an effective tumour-targeting vector.

Recent findings have shown that chimeric antigen receptor (CAR)-T cells can be used to treat tumours expressing chimeric antigen receptors after the gene transformation of B-cell malignant tumours (11). However, due to the limitations of tumour-specific targets, CAR-T cell therapy is ineffective in patients with solid tumours (11). CLDN6 is a surface antigen of carcinoembryonic cells and has an ideal expression profile for CAR-T cells. Although some research conclusions are inconsistent on this topic, study results show that CLDN6 LPX can cause CLDN6-CAR-T proliferation and survival effectively in vivo, allowing CAR-T cells to treat solid tumours (73). This provides a new strategy for the treatment of solid tumours using CAR-T cells.

In addition, it has been found that CLDN6-mediated measles virus (MV) can induce tumour-associated antigen-specific humoral immunity. Mice inoculated with the CLDN6 vaccine showed targeted complement-dependent cytotoxicity with cytolytic activity. MV combined with a tumour lytic effect and the CLDN6 tumour vaccine was able to break the tolerance to endogenous tumour antigens and produce a highly effective and specific antitumour immune response (74). These studies suggest that CLDN6 may provide a new strategy for tumour vaccine treatments of solid tumours.

\section{Discussion}

Reinhard et al (11) believed that CLDN6 is a strictly carcinoembryonic antigen that is expressed exclusively in tumour tissues. However, the present study reviewed the expression of CLDN6 in various tumours and found that the research conclusions for several tumour types were not completely consistent or were contradictory. Possible reasons for inconsistent conclusions include heterogeneity in tumours, use of monoclonal antibodies, different experimental methods and the interpretation of the results. In addition, the role of CLDN6 differs by tumour type, as it promotes some tumours, inhibits others and shows inconsistent (promotional and inhibitory) effects in some tumours. 
However, in liver, ovarian, endometrial and oesophageal cancer types, the expression of CLDN6 is consistently expressed in tumour tissues, compared with no or less expression in the surrounding normal tissues. Therefore, CLDN6 has potential as a carcinoembryonic antigen and a therapeutic target for these tumours.

The reason for CLDN6 expression being upregulated in some tumours and downregulated in others remains unclear. Possible reasons include detection errors or CLDN6 having different roles in different tumours. This area requires further research. Moreover, currently, there are no studies of whether a relationship exists between these tumours; this needs to be further explored. The expression of other CLDNs and their synergistic effects with CLDN6 have been reported. In breast cancer, overexpression of CLDN3, -4 and -7 was primarily dependent on oestrogen receptor-status, whereas overexpression of CLDN6 and high membranous expression of CLDN1 were independent of other characteristics (24). Another study showed that CLDN5, CLDN9, CLDN12 and CLDN13 were not expressed in breast carcinoma tissues or non-neoplastic tissues (22). In addition, CLDN1, CLDN3, CLDN8 and CLDN10 were expressed in breast carcinoma and non-neoplastic tissues, but there was no significant difference between the expression of all these CLDN proteins. The expression of CLDN2, CLDN6 and CLDN14 was downregulated, while the expression of CLDN11 was upregulated in breast carcinoma compared with those in non-neoplastic tissues. In addition, a distinct prognostic significance in the expression of CLDN3 and mostly of CLDN4 between triple-negative and luminal breast carcinomas was identified (75). In gastric cancer, the expression of CLDN2 and CLDN6 was downregulated in gastric cancer tissue, while the expression of CLDN11 was upregulated (36). CLDN6 induces MMP2 activation through CLDN1 membrane expression, which in turn promotes cell migration and invasiveness (44). CLDN6 expression was high in both intestinal- and diffuse-type gastric adenocarcinomas. CLDN7 was expressed mainly in the diffuse-type, whereas CLDN9 was primarily found in the apical membrane of the gland cells in the intestinal-type (40). In addition, CLDN7, -3 and -4 have been detected in gastric cancer (76-78).

CLDN6 has more than one regulatory mechanism in a variety of tumours, such as breast and gastric cancer. These mechanisms may work synergistically. However, the primary mechanism, as well as the effect(s) of different regulatory mechanisms, remain to be determined and require further study.

Although there are still a number of controversies regarding the expression and regulation mechanisms of CLDN6, CLDN6 is a potential antigen and has the potential to be used as a target for a variety of tumours and treatments.

\section{Conclusion}

CLDN6 is expressed in a variety of tumours, and its expression levels differ by tumour type. In some tumours, research consistently shows that CLDN6 is expressed in tumour tissues, but is not expressed or expressed at low levels in surrounding tissues. These include liver, ovarian, endometrial and oesophageal cancer, and AT/RTs, among others. In these tumours, CLDN6 has potential as a carcinoembryonic antigen and a therapeutic target. However, in other tumours, the expression levels of CLDN6 between tumour and surrounding tissues differ from one another or are even the opposite. These include breast, gastric, cervical and lung cancer. In the future, CLDN6 may be used as a target for a variety of therapeutic types such as antibody-drug radionuclide-targeted drugs and ADCs.

\section{Acknowledgements}

Not applicable.

\section{Funding}

This study was supported by the Mianyang Science and Technology Bureau (grant no. 15-S01-3).

\section{Availability of data and materials}

Not applicable.

\section{Authors' contributions}

$\mathrm{XD}$ contributed to the conception and design of this study. HD, XY and JF searched for relevant literature. HD wrote the manuscript. XD provided advice and was responsible for revising the manuscript. All authors read and approved the final version of the manuscript. Data authentication is not applicable.

\section{Ethics approval and consent to participate}

Not applicable.

\section{Patient consent for publication}

Not applicable.

\section{Competing interests}

The authors declare that they have no competing interests.

\section{References}

1. Li J, Ananthapanyasut $\mathrm{W}$ and Yu AS: Claudins in renal physiology and disease. Pediatr Nephrol 26: 2133-2142, 2011.

2. Singh AB, Uppada SB and Dhawan P: Claudin proteins, outside-in signaling, and carcinogenesis. Pflugers Arch 469: 69-75, 2017.

3. Hu CA, Hou Y, Yi D, Qiu Y, Wu G, Kong X and Yin Y: Autophagy and tight junction proteins in the intestine and intestinal diseases. Anim Nutr 1: 123-127, 2015.

4. English DP and Santin AD: Claudins overexpression in ovarian cancer: Potential targets for clostridium perfringens enterotoxin (CPE) based diagnosis and therapy. Int J Mol Sci 14: 10412-10437, 2013.

5. Tabariès S and Siegel PM: The role of claudins in cancer metastasis. Oncogene 36: 1176-1190, 2017

6. Ushiku T, Shinozaki-Ushiku A, Maeda D, Morita S and Fukayama M: Distinct expression pattern of claudin-6, a primitive phenotypic tight junction molecule, in germ cell tumours and visceral carcinomas. Histopathology 61: 1043-1056, 2012.

7. Gowrikumar S, Singh AB and Dhawan P: Role of claudin proteins in regulating cancer stem cells and chemoresistance-potential implication in disease prognosis and therapy. Int J Mol Sci 21: 53, 2019. 
8. Kwon MJ: Emerging roles of claudins in human cancer. Int J Mol Sci 14: 18148-18180, 2013.

9. Singh AB and Dhawan P: Claudins and cancer: Fall of the soldiers entrusted to protect the gate and keep the barrier intact. Semin Cell Dev Biol 42: 58-65, 2015.

10. Chen S, Liu X and Luo W: Advances in the application of claudins to tumor therapy. Sheng Wu Gong Cheng Xue Bao 35: 931-941, 2019 (In Chinese).

11. Reinhard K, Rengstl B, Oehm P, Michel K, Billmeier A, Hayduk N, Klein O, Kuna K, Ouchan Y, Wöll S, et al: An RNA vaccine drives expansion and efficacy of claudin-CAR-T cells against solid tumors. Science 367: 446-453, 2020.

12. Singh AB, Sharma A and Dhawan P: Claudin family of proteins and cancer: An overview. J Oncol 2010: 541957, 2010.

13. Lin D, Guo Y, Li Y, Ruan Y, Zhang M, Jin X, Yang M, Lu Y, Song P, Zhao S, et al: Bioinformatic analysis reveals potential properties of human claudin-6 regulation and functions. Oncol Rep 38: 875-885, 2017.

14. Anderson WJ, Zhou Q, Alcalde V, Kaneko OF, Blank LJ, Sherwood RI, Guseh JS, Rajagopal J and Melton DA: Genetic targeting of the endoderm with claudin-6CreER. Dev Dyn 237 504-512, 2008

15. Sugimoto K, Ichikawa-Tomikawa N, Kashiwagi K, Endo C, Tanaka S, Sawada N, Watabe T, Higashi T and Chiba H: Cell adhesion signals regulate the nuclear receptor activity. Proc Natl Acad Sci USA 116: 24600-24609, 2019.

16. Hoevel T, Macek R, Swisshelm K and Kubbies M: Reexpression of the TJ protein CLDN1 induces apoptosis in breast tumor spheroids. Int J Cancer 108: 374-383, 2004.

17. Yafang L, Qiong W, Yue R, Xiaoming X, Lina Y, Mingzi Z, Ting Z, Yulin L and Chengshi Q: Role of estrogen receptor- $\alpha$ in the regulation of claudin-6 expression in breast cancer cells J Breast Cancer 14: 20-27, 2011.

18. Mullin JM: Potential interplay between luminal growth factors and increased tight junction permeability in epithelial carcinogenesis. J Exp Zool 279: 484-489, 1997.

19. Stadler CR, Bähr-Mahmud H, Plum LM, Schmoldt K, Kölsch AC Türeci Ö and Sahin U: Characterization of the first-in-class T-cell-engaging bispecific single-chain antibody for targeted immunotherapy of solid tumors expressing the oncofetal protein claudin 6. Oncoimmunology 5: e1091555, 2015.

20. Ben-David U, Nudel $\mathrm{N}$ and Benvenisty $\mathrm{N}$ : Immunologic and chemical targeting of the tight-junction protein claudin- 6 eliminates tumorigenic human pluripotent stem cells. Nat Commun 4: 1992, 2013

21. Wu Q, Liu Y, Ren Y, Xu X, Yu L, Li Y and Quan C: Tight junction protein, claudin-6, downregulates the malignant phenotype of breast carcinoma. Eur J Cancer Prev 19: 186-194, 2010.

22. Jia H, Chai X, Li S, Wu D and Fan Z: Identification of claudin-2, $-6,-11$ and -14 as prognostic markers in human breast carcinoma. Int J Clin Exp Pathol 12: 2195-2204, 2019.

23. Wu Q, Liu YF, Ren Y, Xu XM, Yu LN, Li YL and Quan CS Effects of stable up-regulation of tight junction protein claudin-6 upon biological phenotypes of breast cancer cell MCF-7. Zhonghua Yi Xue Za Zhi 90: 407-412, 2010 (In Chinese).

24. Heerma van Voss MR, van Diest PJ, Smolders YH, Bart J, van der Wall E and van der Groep P: Distinct claudin expression characterizes BRCA1-related breast cancer. Histopathology 65 814-827, 2014

25. Xu X, Jin H, Liu Y, Liu L, Wu Q, Guo Y, Yu L, Liu Z, Zhang T, Zhang $\mathrm{X}$, et al: The expression patterns and correlations of claudin-6, methy-CpG binding protein 2, DNA methyltransferase 1, histone deacetylase 1, acetyl-histone $\mathrm{H} 3$ and acetyl-histone $\mathrm{H} 4$ and their clinicopathological significance in breast invasive ductal carcinomas. Diagn Pathol 7: 33, 2012.

26. Lu Y, Wang L, Li H, Li Y, Ruan Y, Lin D, Yang M, Jin X, Guo Y, Zhang $X$ and Quan C: SMAD2 inactivation inhibits CLDN6 methylation to suppress migration and invasion of breast cancer cells. Int J Mol Sci 18: 1863, 2017.

27. Li Q, Zhu F and Chen P: miR-7 and miR-218 epigenetically control tumor suppressor genes RASSF1A and claudin-6 by targeting HoxB3 in breast cancer. Biochem Biophys Res Commun 424: 28-33, 2012.

28. Liu Y, Jin X, Li Y, Ruan Y, Lu Y, Yang M, Lin D, Song P, Guo Y, Zhao S, et al: DNA methylation of claudin-6 promotes breast cancer cell migration and invasion by recruiting MeCP2 and deacetylating H3Ac and H4Ac. J Exp Clin Cancer Res 35: 120,2016
29. Ren Y, Wu Q, Liu Y, Xu X and Quan C: Gene silencing of claudin- 6 enhances cell proliferation and migration accompanied with increased MMP-2 activity via p38 MAPK signaling pathway in human breast epithelium cell line HBL 100. Mol Med Rep 8: 1505-1510, 2013.

30. Jia Y, Guo Y, Jin Q, Qu H, Qi D, Song P, Zhang X, Wang X, Xu W, Dong Y, et al: A SUMOylation-dependent HIF-1 $\alpha /$ CLDN6 negative feedback mitigates hypoxia-induced breast cancer metastasis. J Exp Clin Cancer Res 39: 42, 2020.

31. Guo Y, Xu X, Liu Z, Zhang T, Zhang X, Wang L, Wang M, Liu Y, Lu Y, Liu Y and Quan C: Apoptosis signal-regulating kinase 1 is associated with the effect of claudin- 6 in breast cancer. Diagn Pathol 7: 111, 2012.

32. Guo Y, Lin D, Zhang M, Zhang X, Li Y, Yang R, Lu Y, Jin X, Yang M, Wang M, et al: CLDN6-induced apoptosis via regulating ASK1-p38/JNK signaling in breast cancer MCF-7 cells. Int J Oncol 48: 2435-2444, 2016.

33. Song P, Li Y, Dong Y, Liang Y, Qu H, Qi D, Lu Y, Jin X, Guo Y, Jia Y, et al: Estrogen receptor $\beta$ inhibits breast cancer cells migration and invasion through CLDN6-mediated autophagy. J Exp Clin Cancer Res 38: 354, 2019.

34. Figueiredo NB, Cestari SH, Conde SJ, Luvizotto RA, De Sibio MT, Perone D, Katayama ML, Carraro DM, Brentani HP, Brentani MM and Nogueira CR: Estrogen-responsive genes overlap with triiodothyronine-responsive genes in a breast carcinoma cell line. ScientificWorldJournal 2014: 969404 , 2014.

35. Wu Q, Liu X, Liu YF, Lu Y, Wang LP, Zhang XW, Li YL and Quan CS: Inhibition of p38 activity reverses claudin-6 induced cell apoptosis, invasion, and migration. Chin Med J (Engl) 126: 3539-3544, 2013

36. Lin Z, Zhang X, Liu Z, Liu Q, Wang L, Lu Y, Liu Y, Wang M, Yang M, Jin X and Quan C: The distinct expression patterns of claudin-2, -6 , and -11 between human gastric neoplasms and adjacent non-neoplastic tissues. Diagn Pathol 8: 133, 2013.

37. Zavala-Zendejas VE, Torres-Martinez AC, Salas-Morales B, Fortoul TI, Montaño LF and Rendon-Huerta EP: Claudin-6, 7, or 9 overexpression in the human gastric adenocarcinoma cell line AGS increases its invasiveness, migration, and proliferation rate. Cancer Invest 29: 1-11, 2011.

38. Kohmoto T, Masuda K, Shoda K, Takahashi R, Ujiro S, Tange S, Ichikawa D, Otsuji E and Imoto I: Claudin- 6 is a single prognostic marker and functions as a tumor-promoting gene in a subgroup of intestinal type gastric cancer. Gastric Cancer 23: 403-417, 2020.

39. Gao F, Li M, Xiang R, Zhou X, Zhu L and Zhai Y: Expression of CLDN6 in tissues of gastric cancer patients: Association with clinical pathology and prognosis. Oncol Lett 17: 4621-4625, 2019.

40. Rendón-Huerta E, Teresa F, Teresa GM, Xochitl GS Georgina AF, Veronica ZZ and Montaño LF: Distribution and expression pattern of claudins 6,7 , and 9 in diffuse- and intestinal-type gastric adenocarcinomas. J Gastrointest Cancer 41: 52-59, 2010.

41. Lu YZ, Li Y,Zhang T and Han ST: Claudin-6 is down-regulated in gastric cancer and its potential pathway. Cancer Biomark 28: 329-340, 2020

42. Resnick MB, Gavilanez M,Newton E, Konkin T, Bhattacharya B, Britt DE, Sabo E and Moss SF: Claudin expression in gastric adenocarcinomas: A tissue microarray study with prognostic correlation. Hum Pathol 36: 886-892, 2005.

43. Yu S, Zhang Y, Li Q, Zhang Z, Zhao G and Xu J: CLDN6 promotes tumor progression through the YAP1-snaill axis in gastric cancer. Cell Death Dis 10: 949, 2019.

44. Torres-Martínez AC, Gallardo-Vera JF, Lara-Holguin AN Montaño LF and Rendón-Huerta EP: Claudin-6 enhances cell invasiveness through claudin-1 in AGS human adenocarcinoma gastric cancer cells. Exp Cell Res 350: 226-235, 2017.

45. Chavarría-Velázquez CO, Torres-Martínez AC, Montaño LF and Rendón-Huerta EP: TLR2 activation induced by $H$. pylori LPS promotes the differential expression of claudin-4, $-6,-7$ and -9 via either STAT 3 and ERK1/2 in AGS cells. Immunobiology 223: 38-48, 2018

46. Zhu ZX, Huang JW, Liao MH and Zeng Y: Treatment strategy for hepatocellular carcinoma in China: Radiofrequency ablation versus liver resection. Jpn J Clin Oncol 46: 1075-1080, 2016.

47. Zheng A, Yuan F, Li Y, Zhu F, Hou P, Li J, Song X, Ding M and Deng H: Claudin-6 and claudin-9 function as additional coreceptors for hepatitis C virus. J Virol 81: 12465-12471, 2007. 
48. Osanai M, Takasawa A, Murata M and Sawada N: Claudins in cancer: Bench to bedside. Pflugers Arch 469: 55-67, 2017.

49. Huang L, Zhao C, Sun K, Yang D, Yan L, Luo D, He J, Hu X, Wang R, Shen X, et al: Downregulation of CLDN6 inhibits cell proliferation, migration, and invasion via regulating EGFR/AKT/mTOR signalling pathway in hepatocellular carcinoma. Cell Biochem Funct 38: 541-548, 2020.

50. Zhang X, Ruan Y, Li Y, Lin D and Quan C: Tight junction protein claudin-6 inhibits growth and induces the apoptosis of cervical carcinoma cells in vitro and in vivo. Med Oncol 32: 148, 2015.

51. Zhang X, Ruan Y, Li Y, Lin D, Liu Z and Quan C: Expression of apoptosis signal-regulating kinase 1 is associated with tight junction protein claudin-6 in cervical carcinoma. Int J Clin Exp Pathol 8: 5535-5541, 2015.

52. Micke P, Mattsson JS, Edlund K, Lohr M, Jirström K, Berglund A, Botling J, Rahnenfuehrer J, Marincevic M, Pontén F, et al: Aberrantly activated claudin 6 and 18.2 as potential therapy targets in non-small-cell lung cancer. Int J Cancer 135: 2206-2214, 2014

53. Wang Q, Zhang Y, Zhang T, Han ZG and Shan L: Low claudin-6 expression correlates with poor prognosis in patients with non-small cell lung cancer. Onco Targets Ther 8: 1971-1977, 2015.

54. Wang L, Jin X, Lin D, Liu Z, Zhang X, Lu Y, Liu Y, Wang M, Yang M, Li J and Quan C: Clinicopathologic significance of claudin-6, occludin, and matrix metalloproteinases-2 expression in ovarian carcinoma. Diagn Pathol 8: 190, 2013.

55. Cao X, He GZ: Knockdown of CLDN6 inhibits cell proliferation and migration via PI3K/AKT/mTOR signaling pathway in endometrial carcinoma cell line HEC-1-B. Onco Targets Ther 11: 6351-6360, 2018.

56. Birks DK, Kleinschmidt-DeMasters BK, Donson AM, Barton VN, McNatt SA, Foreman NK and Handler MH: Claudin 6 is a positive marker for atypical teratoid/rhabdoid tumors Brain Pathol 20: 140-150, 2010.

57. Sullivan LM, Yankovich T, Le P, Martinez D, Santi M, Biegel JA Pawel BR and Judkins AR: Claudin-6 is a nonspecific marker for malignant rhabdoid and other pediatric tumors. Am J Surg Pathol 36: 73-80, 2012

58. Antonelli M, Hasselblatt M, Haberler C, Di Giannatale A, Garrè ML, Donofrio V, Lauriola L, Ridola V, Arcella A, Frühwald $M$ and Giangaspero F: Claudin- 6 is of limited sensitivity and specificity for the diagnosis of atypical teratoid/rhabdoid tumors. Brain Pathol 21: 558-563, 2011.

59. Tsunoda S, Smith E, De Young NJ, Wang X, Tian ZQ, Liu JF, Jamieson GG and Drew PA: Methylation of CLDN6, FBN2, RBP1, RBP4, TFPI2, and TMEFF2 in esophageal squamous cell carcinoma. Oncol Rep 21: 1067-1073, 2009.

60. Phi LTH, Sari IN, Yang YG, Lee SH, Jun N, Kim KS, Lee YK and Kwon HY: Cancer stem cells (CSCs) in drug resistance and their therapeutic implications in cancer treatment. Stem Cells Int 2018: 5416923, 2018.

61. Abdullah LN and Chow EK: Mechanisms of chemoresistance in cancer stem cells. Clin Transl Med 2: 3, 2013.

62. Wang L, Xue Y, Shen Y, Li W, Cheng Y, Yan X, Shi W, Wang J, Gong Z, Yang G, et al: Claudin 6: A novel surface marker for characterizing mouse pluripotent stem cells. Cell Res 22 1082-1085, 2012.
63. Turksen K and Troy TC: Claudin-6: A novel tight junction molecule is developmentally regulated in mouse embryonic epithelium. Dev Dyn 222: 292-300, 2001.

64. Gonzalez-Angulo AM, Morales-Vasquez F and Hortobagyi GN: Overview of resistance to systemic therapy in patients with breast cancer. Adv Exp Med Biol 608: 1-22, 2007.

65. Yang M, Li Y, Shen X, Ruan Y, Lu Y, Jin X, Song P, Guo Y, Zhang $\mathrm{X}, \mathrm{Qu} \mathrm{H}$, et al: CLDN6 promotes chemoresistance through GSTP1 in human breast cancer. J Exp Clin Cancer Res 36: 157, 2017.

66. Yang M, Li Y, Ruan Y, Lu Y, Lin D, Xie Y, Dong B, Dang Q and Quan C: CLDN6 enhances chemoresistance to ADM via AF-6/ERKs pathway in TNBC cell line MDAMB231. Mol Cell Biochem 443: 169-180, 2018.

67. Chieffi P, De Martino M and Esposito F: New anti-cancer strategies in testicular germ cell tumors. Recent Pat Anticancer Drug Discov 14: 53-59, 2019.

68. Thomas A, Teicher BA and Hassan R: Antibody-drug conjugates for cancer therapy. Lancet Oncol 17: e254-e262, 2016.

69. Birrer MJ, Moore KN, Betella I and Bates RC: Antibody-drug conjugate-based therapeutics: State of the science. J Natl Cancer Inst 111: 538-549, 2019.

70. Tsuchikama K and An Z: Antibody-drug conjugates: Recent advances in conjugation and linker chemistries. Protein Cell 9: 33-46, 2018.

71. Gudkov SV, Shilyagina NY, Vodeneev VA and Zvyagin AV: Targeted radionuclide therapy of human tumors. Int J Mol Sci 17: 33, 2015.

72. Gill MR, Falzone N, Du Y and Vallis KA: Targeted radionuclide therapy in combined-modality regimens. Lancet Oncol 18: e414-e423, 2017.

73. Schneider IC, Hartmann J, Braun G, Stitz J, Klamp T, Bihi M, Sahin U and Buchholz CJ: Displaying tetra-membrane spanning claudins on enveloped virus-like particles for cancer immunotherapy. Biotechnol J 13: e1700345, 2018.

74. Hutzler S, Erbar S, Jabulowsky RA, Hanauer JRH, Schnotz JH, Beissert T, Bodmer BS, Eberle R, Boller K, Klamp T, et al: Antigen-specific oncolytic MV-based tumor vaccines through presentation of selected tumor-associated antigens on infected cells or virus-like particles. Sci Rep 7: 16892, 2017.

75. Kolokytha P, Yiannou P, Keramopoulos D, Kolokythas A, Nonni A, Patsouris E and Pavlakis K: Claudin-3 and claudin-4: Distinct prognostic significance in triple-negative and luminal breast cancer. Appl Immunohistochem Mol Morphol 22: 125-131, 2014.

76. Wu Z, Shi J, Song Y, Zhao J, Sun J, Chen X, Gao P and Wang Z: Claudin-7 (CLDN7) is overexpressed in gastric cancer and promotes gastric cancer cell proliferation, invasion and maintains mesenchymal state. Neoplasma 65: 349-359, 2018.

77. Danilova NV, Anikina KA, Oleynikova NA, Vychuzhanin DV and Malkov PG: Claudin-3 expression in gastric cancer. Arkh Patol 82: 5-11, 2020 (In Russian).

78. Liu JX, Wei ZY, Chen JS, Lu HC, Hao L and Li WJ: Prognostic and clinical significance of claudin-4 in gastric cancer: A meta-analysis. World J Surg Oncol 13: 207, 2015.

This work is licensed under a Creative Commons Attribution-NonCommercial-NoDerivatives 4.0 International (CC BY-NC-ND 4.0) License. 